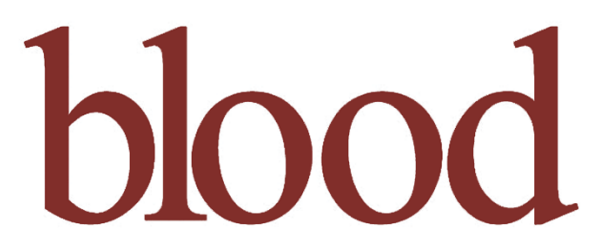

2008 112: 1005-1012

Prepublished online May 13, 2008;

doi:10.1182/blood-2008-02-140665

\title{
Dasatinib crosses the blood-brain barrier and is an efficient therapy for central nervous system Philadelphia chromosomepositive leukemia
}

Kimmo Porkka, Perttu Koskenvesa, Tuija Lundán, Johanna Rimpiläinen, Satu Mustjoki, Richard Smykla, Robert Wild, Roger Luo, Montserrat Arnan, Benoit Brethon, Lydia Eccersley, Henrik Hjorth-Hansen, Martin Höglund, Hana Klamova, Håvar Knutsen, Suhag Parikh, Emmanuel Raffoux, Franz Gruber, Finella Brito-Babapulle, Hervé Dombret, Rafael F. Duarte, Erkki Elonen, Ron Paquette, C. Michel Zwaan and Francis Y. F. Lee

Updated information and services can be found at:

http://bloodjournal.hematologylibrary.org/cgi/content/full/112/4/1005

Articles on similar topics may be found in the following Blood collections:

Neoplasia (4222 articles)

Free Research Articles (647 articles)

Clinical Trials and Observations (2585 articles)

Information about reproducing this article in parts or in its entirety may be found online at:

http://bloodjournal.hematologylibrary.org/misc/rights.dtl\#repub_requests

Information about ordering reprints may be found online at:

http://bloodjournal.hematologylibrary.org/misc/rights.dt|\#reprints

Information about subscriptions and ASH membership may be found online at:

http://bloodjournal.hematologylibrary.org/subscriptions/index.dtl

Blood (print ISSN 0006-4971, online ISSN 1528-0020), is published semimonthly by the American Society of Hematology, 1900 M St, NW, Suite 200, Washington DC 20036.

Copyright 2007 by The American Society of Hematology; all rights reserved.

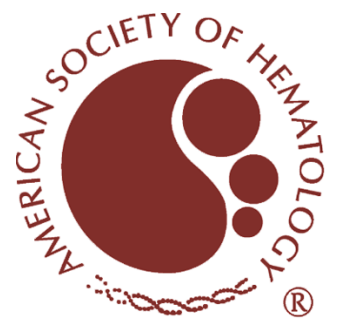




\title{
Dasatinib crosses the blood-brain barrier and is an efficient therapy for central nervous system Philadelphia chromosome-positive leukemia
}

\author{
Kimmo Porkka, ${ }^{1,2}$ Perttu Koskenvesa, ${ }^{1,2}$ Tuija Lundán, ${ }^{1,3}$ Johanna Rimpiläinen, ${ }^{4}$ Satu Mustjoki, ${ }^{1,2}$ Richard Smykla, ${ }^{5}$ \\ Robert Wild, ${ }^{6}$ Roger Luo, ${ }^{5}$ Montserrat Arnan, ${ }^{7}$ Benoit Brethon, ${ }^{8}$ Lydia Eccersley, ${ }^{9}$ Henrik Hjorth-Hansen, ${ }^{10}$ Martin Höglund, ${ }^{11}$ \\ Hana Klamova, ${ }^{12}$ Håvar Knutsen, ${ }^{13}$ Suhag Parikh, ${ }^{14}$ Emmanuel Raffoux, ${ }^{15}$ Franz Gruber, ${ }^{16}$ Finella Brito-Babapulle, ${ }^{9}$ \\ Hervé Dombret, ${ }^{15}$ Rafael F. Duarte, ${ }^{7}$ Erkki Elonen, ${ }^{2}$ Ron Paquette, ${ }^{17}$ C. Michel Zwaan, ${ }^{18,19}$ and Francis Y. F. Lee ${ }^{5}$ \\ ${ }^{1}$ Hematology Research Unit, Biomedicum Helsinki, Helsinki, Finland; ${ }^{2}$ Departments of Clinical Chemistry and Medicine/Hematology, and ${ }^{3} \mathrm{HUSLAB}$, Department \\ of Molecular Pathology, Helsinki University Central Hospital, Helsinki, Finland; ${ }^{4}$ North-Karelia Central Hospital, Joensuu, Finland; ${ }^{5}$ Bristol-Myers Squibb \\ Research and Development, Princeton, NJ; ${ }^{\circ}$ OSI Pharmaceuticals, In Vivo Pharmacology, Boulder, CO; ${ }^{7}$ ICO-Hospital Duran i Reynals, Barcelona, Spain; \\ ${ }^{8}$ Hôpital Saint-Louis, Pediatric Hematology, Paris, France; ${ }^{9}$ Ealing Hospital, Southall, United Kingdom; ${ }^{10}$ Department of Hematology, St Olavs Hospital and \\ Department of Molecular Medicine and Cancer Research, Norwegian University of Science and Technology, Trondheim, Norway; ${ }^{11}$ Uppsala University Hospital, \\ Uppsala, Sweden; ${ }^{12}$ Institute of Hematology and Blood Transfusion, Prague, Czech Republic; ${ }^{13}$ Akershus University Hospital, Lorenskog, Norway; ${ }^{14}$ Duke \\ University Medical Center, Pediatric Blood and Marrow Transplant Program, Durham, NC; ${ }^{15}$ Hôpital Saint-Louis, Paris, France; ${ }^{16}$ University of Troms $\varnothing$, Troms $\varnothing$, \\ Norway; ${ }^{17}$ University of California-Los Angeles; ${ }^{18}$ Erasmus Medical Center (MC), Pediatric Oncology, Rotterdam, The Netherlands; and ${ }^{19}$ The Innovative \\ Therapies for Children with Cancer (ITCC) Consortium, Paris, France
}

\begin{abstract}
Although imatinib, a BCR-ABL tyrosine kinase inhibitor, is used to treat acute Philadelphia chromosome-positive $\left(\mathrm{Ph}^{+}\right)$ leukemia, it does not prevent central nervous system (CNS) relapses resulting from poor drug penetration through the blood-brain barrier. Imatinib and dasatinib (a dual-specific SRC/BCR-ABL kinase inhibitor) were compared in a preclinical mouse model of intracranial $\mathrm{Ph}^{+}$ leukemia. Clinical dasatinib treatment in patients with $\mathrm{CNS} \mathrm{Ph}^{+}$leukemia was assessed. In preclinical studies, dasatinib
\end{abstract}

increased survival, whereas imatinib failed to inhibit intracranial tumor growth. Stabilization and regression of CNS disease were achieved with continued dasatinib administration. The drug also demonstrated substantial activity in 11 adult and pediatric patients with $\mathrm{CNS} \mathrm{Ph}^{+}$leukemia. Eleven evaluable patients had clinically significant, long-lasting responses, which were complete in 7 patients. In 3 additional patients, isolated CNS relapse occurred during dasatinib therapy; and in 2 of them, it was caused by expan- sion of a $B C R-A B L-$ mutated dasatinibresistant clone, implying selection pressure exerted by the compound in the CNS. Dasatinib has promising therapeutic potential in managing intracranial leukemic disease and substantial clinical activity in patients who experience CNS relapse while on imatinib therapy. This study is registered at ClinicalTrials. gov as CA180006 (\#NCT00108719) and CA180015 (\#NCT00110097). (Blood. 2008; 112:1005-1012)

\section{Introduction}

Central nervous system (CNS) involvement is a relatively common complication in Philadelphia chromosome $(\mathrm{Ph})$-positive acute lymphoblastic leukemia $\left(\mathrm{Ph}^{+} \mathrm{ALL}\right)$ and blast crisis-chronic myelogenous leukemia (BC-CML). ${ }^{1}$ Imatinib is a first-generation BCRABL tyrosine kinase inhibitor, which is approved for use in patients with $\mathrm{Ph}^{+}$leukemias. Despite its use, up to $20 \%$ of imatinib-treated patients with either lymphoid or myeloid $\mathrm{BC}-\mathrm{CML}$ or $\mathrm{Ph}^{+} \mathrm{ALL}$ develop CNS relapses. ${ }^{1,2}$ In many cases, these have occurred despite complete responses in peripheral blood and bone marrow ${ }^{2-4}$ and are attributable to poor penetration of imatinib into the cerebrospinal fluid (CSF) with inadequate concentrations for kinase inhibition. ${ }^{5}$ This may be, at least partly, because imatinib is a substrate for the drug-eluting P-glycoprotein, which results in subtherapeutic levels of imatinib in the CNS. ${ }^{1,5-8}$

Conventional therapy for CNS leukemia, including intrathecal chemotherapy, high-dose systemic chemotherapy (cytarabine, methotrexate), and radiotherapy, is unsatisfactory as many patients experience significant toxicity, short-lived responses, and ultimately death resulting from refractory leukemia. ${ }^{9}$

Dasatinib, a second-generation inhibitor of tyrosine kinases SRC and BCR-ABL, has shown significant activity in adults with imatinib-resistant or -intolerant CML or $\mathrm{Ph}^{+}$ALL. ${ }^{10-14}$ In vitro, dasatinib has 325 -fold greater potency than imatinib for inhibiting BCR-ABL. ${ }^{15}$ It effectively inhibits the growth of leukemic clones harboring all known imatinib-resistant $B C R$ $A B L$ kinase domain point mutations, with the exception of V299L, T315I, and F317L. ${ }^{16}$ Dasatinib also has antileukemic activity in the multidrug-resistant K562/ADM model that highly expresses the P-glycoprotein drug efflux pump. ${ }^{17}$ A recent single case report suggested regression of $\mathrm{Ph}^{+} \mathrm{CNS}$ leukemia after dasatinib therapy. ${ }^{18}$

Based on these results, we further investigated the preclinical and clinical effects of dasatinib in CNS leukemia. We show that dasatinib is associated with increased survival in a mouse model of
Submitted February 19, 2008; accepted April 14, 2008. Prepublished online as Blood First Edition paper, May 13, 2008; DOI 10.1182/blood-2008-02-140665.

The online version of this article contains a data supplement.
The publication costs of this article were defrayed in part by page charge payment. Therefore, and solely to indicate this fact, this article is hereby marked "advertisement" in accordance with 18 USC section 1734.

@ 2008 by The American Society of Hematology 
intracranial CML and induces clinically meaningful responses in patients with CNS disease.

\section{Methods}

\section{Preclinical analysis of dasatinib in an animal model of intracranial CML}

Compounds, tumor cell lines, and reagents. Dasatinib (synthesized by Bristol-Myers Squibb, Princeton, NJ) and commercially available imatinib (Novartis, Basel, Switzerland) were dissolved at $10 \mathrm{mM}$ in $100 \%$ dimethyl sulfoxide (DMSO). In the in vitro experiments, compounds were diluted with culture medium to yield final concentrations of less than or equal to $0.1 \%$ DMSO. Sterile buffers and solutions were obtained from Invitrogen (Carlsbad, CA). Sterile tissue culture ware was obtained from Fisher Scientific (Pittsburgh, PA). K562 CML cells were obtained from ATCC (Manassas, VA) and maintained in fetal bovine serum (Invitrogen).

Study protocols were approved by the Institutional Review Board/ Independent Ethics Committee at each participating center.

In vitro cell proliferation assay. K562 CML cells were seeded at $10^{5}$ cells $/ \mathrm{mL}$ in regular culture medium into 6-well plates, and different concentrations of compound (or DMSO for controls) were added. At 24, 48, and 72 hours after treatment, cells were harvested and total viable cell counts were determined using the ViCell cell viability analyzer (Beckman Coulter, Fullerton, CA).

Transfection of $\mathbf{K 5 6 2}$ cells with luciferase construct. The firefly luciferase (luc) gene was subcloned from pGL3 (Promega, Madison, WI) into the pcDNA6.2 expression vector (Invitrogen). Transfection of the K562 cell line was performed using Lipofectamine 2000 reagent (Invitrogen), and cells were selected with $10 \mu \mathrm{g} / \mathrm{mL}$ Blasticidin (Invitrogen) for 2 weeks. Single colonies were cloned using a soft agar colony formation assay. Individual clones were then screened for bioluminescence using an in vitro luciferase assay (LucLite, PerkinElmer Life and Analytical Sciences, Waltham, MA). Bioluminescence of individual clones was verified in vitro using the IVIS Imaging System (Xenogen, Alameda, CA). Initially, 15 antibiotic-resistant clones were isolated from the soft agar assay. Clone K562-pLUC\#2 (Figure S1, available on the Blood website; see the Supplemental Materials link at the top of the online article) was eventually selected for use because it exhibited the following desirable characteristics: stable luminescence expression, sensitivity to BCR-ABL inhibitors comparable with the parental K562 cells, and high in vivo tumorigenicity.

Animals. Female severe combined immunodeficient (SCID)-beige mice, 5 to 6 weeks of age, were obtained from Harlan (Indianapolis, IN) and maintained in an ammonia-free environment in a defined and pathogen-free colony. They were fed food and water ad libitum. All studies were performed in accordance with the American Association for Accreditation of Laboratory Animal Care.

Tumor model and compound administration. Before leukemic cell implantation, animals were anesthetized using Avertin (Sigma-Aldrich, St Louis, MO) administered intraperitoneally, at a dose of $250 \mathrm{mg} / \mathrm{kg}$. K562 cells or bioluminescent K562-pLUC\#2 cells $\left(2 \times 10^{6}\right.$ cells/ mouse) were then injected intracranially in a total volume of $0.02 \mathrm{~mL}$ into SCID-beige mice.

Five to 9 days after cell implantation, mice were randomized into treatment and control groups, and treatment was initiated. Dasatinib was formulated in propylene glycol:water (1:1). Imatinib was formulated in 5\% carboxymethylcellulose solution. Both compounds were administered orally in a volume of $0.01 \mathrm{~mL} / \mathrm{g}$ of mouse body weight.

Antitumor activity was evaluated in terms of (1) increases in lifespan reflected by the prolongation of median survival time (MST) and expressed as $\% \mathrm{~T} / \mathrm{C}$ value (ie, the ratio of MST of treated vs control groups); and (2) inhibition of tumor growth as determined by bioluminescence imaging (BLI). Activity was defined as a statistically significant increase in lifespan corresponding to a T/C more than or equal to $125 \%$.

Statistical analysis. Statistical significance for survival and tumor growth in the preclinical studies was determined by the Gehan generalized Wilcoxon test and required a $P$ value less than .05 .
Bioluminescence imaging. Bioluminescence signals were analyzed using Living Image software (Caliper Life Sciences, Hopkinton, MA). For in vitro imaging, bioluminescent cells at various concentrations were incubated at $37^{\circ} \mathrm{C}$ with D-luciferin (Xenogen) at $150 \mu \mathrm{g} / \mathrm{mL}$ for 7 to 10 minutes before imaging for 2 minutes. For in vivo imaging, mice were given an intraperitoneal dose of $150 \mathrm{mg} / \mathrm{kg}$ of D-luciferin immediately before being anesthetized with Avertin $(250 \mathrm{mg} / \mathrm{kg})$ and were imaged 15 minutes later using the IVIS Imaging System 100 Series (Xenogen). Quantitation was based on total flux (photons/sec) of emitted light as a measure of the relative number of viable tumor cells. Data were analyzed using Xenogen Living Image software.

Dasatinib plasma and brain pharmacokinetics in mice. Plasma and brain dasatinib concentrations were determined by high performance liquid chromatography/mass spectroscopy (HPLC/MS). The analytical equipment used consisted of a Hewlett Packard (Palo Alto, CA) model 1100 HPLC/Autosampler combination, coupled to a Phenomenex Prodigy C18-ODS3 column. A gradient elution method was used with a mobile phase consisted of $5 \mathrm{mM}$ of ammonium acetate in a mix of water/acetonitrile, $\mathrm{pH}$ 5.0. The HPLC was interfaced to a Finnigan LCQ Advantage ion-trap mass spectrometer (Thermo Fisher Scientifics, Waltham, MA) operated in the positive ion electrospray, full MS/MS mode. For dasatinib, fragmentation of $\mathrm{m} / \mathrm{z} 488$ yielded daughter ions for quantitation at $\mathrm{m} / \mathrm{z} 401$. For the internal standard, $\mathrm{m} / \mathrm{z} 444$ was fragmented to yield daughters at $\mathrm{m} / \mathrm{z} 303$. Helium was the collision gas. The standard curve ranged from $2.7 \mathrm{nM}$ to $40 \mu \mathrm{M}$ and was fitted with a quadratic regression weighted by reciprocal concentration $(1 / \mathrm{x})$. The limit of quantitation was $2.7 \mathrm{nM}$. Plasma samples $(50 \mu \mathrm{L})$ were deproteinized before analysis with 3 volumes of acetonitrile containing $5 \mu \mathrm{g} / \mathrm{mL}$ BMS-354990 as internal standard. Brain samples were homogenized in 2 parts water before dilution in acetonitrile. Plasma/brain ratios were calculated to assess the relative ability of dasatinib to cross the blood-brain barrier.

\section{Clinical analysis of dasatinib in $\mathrm{CNS} \mathrm{Ph}^{+}$leukemia}

Patients. Patients with $\mathrm{Ph}^{+}$ALL or BC-CML and CNS involvement who received treatment with dasatinib, either as part of clinical trials evaluating dasatinib in $\mathrm{Ph}^{+} \mathrm{ALL}$ or BC-CML or on a "compassionate-use" basis, were identified. None of the patients was using dasatinib at the time of CNS disease diagnosis, with the exception of a subgroup of the 3 patients who developed CNS disease relapse while on dasatinib therapy. Informed consent was obtained from all patients taking part in clinical trials in accordance with the Declaration of Helsinki.

Treatment. Adult patients received oral dasatinib $140 \mathrm{mg}$ once daily or $70 \mathrm{mg}$ twice daily. The dasatinib dose could be adjusted after one cycle of treatment (4 weeks), with dose escalation in patients with lack of response and dose reduction in patients with unacceptable toxicity. Pediatric and adolescent patients with $\mathrm{Ph}^{+}$ALL treated according to study protocol received oral dasatinib 60 to $160 \mathrm{mg} / \mathrm{m}^{2}$ once daily. Dasatinib was administered until disease progression, intolerable toxicity, withdrawal of consent, or until the investigator and patient agreed that discontinuation of therapy was in the best interest of the patient.

Patient evaluation. Lumbar punctures were performed every 1 to 4 weeks until resolution to follow outcome. Responses were classified according to criteria described by Odom et al. ${ }^{19}$ Briefly, a complete response was defined by an absence of blasts in the CSF, a very good partial response as a CSF mononuclear cell count less than $5 \times 10^{6} / \mathrm{L}$ and identification of only single blasts on cytospin slide examination, and a partial response as a decrease in CSF mononuclear cell count by more than $50 \%$. Response duration was measured from the first day the response criteria were met until the date treatment was discontinued because of progression or death, or the date of the last CNS assessment. Dasatinib and dasatinib metabolite concentrations within the CSF were measured by HPLC/MS/MS chromatography.

Analysis of BCR-ABL gene mutations in CSF. The total RNA from CSF cell samples was extracted using RNeasy Mini Kit (Qiagen, Hilden, Germany) according to the manufacturer's instructions. Because of the small number of cells resulting in low RNA yields, the samples were subjected to RNA amplification with Agilent Low RNA Input Fluorescent Linear Amplification Kit (Agilent Technologies, Palo Alto, CA). The amplified cRNA was then purified and further reverse-transcribed to cDNA 
From www.bloodjournal.org at Erasmus MC Medical Library on May 20, 2009. For personal use only.

BLOOD, 15 AUGUST 2008 • VOLUME 112, NUMBER 4

Table 1. Antitumor activity of dasatinib and imatinib in intracranial CML models

\begin{tabular}{|c|c|c|c|c|c|}
\hline \multirow{2}{*}{$\begin{array}{l}\text { Tumor (study no.), } \\
\text { compound }\end{array}$} & \multirow[b]{2}{*}{ Dose, $\mathrm{mg} / \mathrm{kg}$} & \multirow{2}{*}{$\begin{array}{c}\text { Route; schedule; } \\
\text { day treatment started }\end{array}$} & \multicolumn{2}{|c|}{ Efficacy $(n=10)$} & \multirow[b]{2}{*}{ Tolerability, AWC, $\mathrm{g}$} \\
\hline & & & MST, days & $\% \mathbf{T} / \mathbf{C}$ & \\
\hline \multicolumn{6}{|l|}{ K562 (1) } \\
\hline Dasatinib & 15 & Orally; BID × 40; 5 & $49.5^{\star}$ & $450 \dagger$ & ND \\
\hline Dasatinib & 5 & Orally; BID × 26; 5 & 29.5 & $268 \dagger$ & ND \\
\hline Imatinib & 100 & Orally; BID $\times 10 ; 5$ & 11.0 & 100 & ND \\
\hline Control & - & & 11.0 & - & ND \\
\hline \multicolumn{6}{|l|}{ K562-pLUC\#2 (2) } \\
\hline Dasatinib & 15 & Orally; BID $\times 14 ; 6$ & 76.0 & $267 \ddagger$ & 1.1 \\
\hline Imatinib & 100 & Orally; BID $\times 14 ; 6$ & 35.0 & 123 & -1.5 \\
\hline Control & - & & 28.5 & - & 1.3 \\
\hline \multicolumn{6}{|l|}{ K562-pLUC\#2 (3) } \\
\hline Dasatinib & 50 & Orally; QD × 30; 9 & 61.5 & $256 \S$ & 0.3 \\
\hline Dasatinib & 25 & Orally; QD × 30; 9 & 55.0 & $229 \S$ & -0.1 \\
\hline Control & - & & 24.0 & - & ND \\
\hline
\end{tabular}

according to published recommendations of Europe Against Cancer program. ${ }^{20}$ Polymerase chain reactions (PCRs) were performed as described with minor modifications. ${ }^{21,22}$ The amplified PCR products were then sequenced with direct sequencing and ABI Prism 3100 Genetic Analyzer (Applied Biosystems, Foster City, CA).

\section{Results}

\section{Antitumor effects of dasatinib and imatinib in mice}

Experiment 1. We initially evaluated the efficacy of dasatinib and imatinib in the K562 intracranial CML tumor model. Drugs were administered orally starting on day 5 after tumor implantation, and treatment was continued until clear disease progression was observed (ie, MST was achieved for each individual treatment set). Dasatinib was administered using a 5-days-on/2-days-off twice-daily schedule (Monday through Friday) at dose levels of $5 \mathrm{mg} / \mathrm{kg}$ and $15 \mathrm{mg} / \mathrm{kg}$, whereas imatinib was administered continuously twice daily (without drug administration break period) at a dose level of $100 \mathrm{mg} / \mathrm{kg}$. Dasatinib was associated with a dose-dependent increase in survival compared with control groups $(P<.01$; Table 1$)$. In contrast, imatinib completely failed to increase the MST.

Experiment 2. We next assessed the antitumor activity of dasatinib and imatinib in the intracranial CML tumor model in more detail. A K562 variant cell line was generated (K562pLUC\#2) overexpressing the firefly luciferase gene to allow noninvasive monitoring of intracranial tumor growth in vivo using BLI. K562-pLUC\#2 cells were injected intracranially into SCIDbeige mice, and the mice treated on day 6 after tumor implantation for 14 days with either dasatinib $15 \mathrm{mg} / \mathrm{kg}$ or imatinib $100 \mathrm{mg} / \mathrm{kg}$ using the same schedules described previously. Tumor growth was measured on day 5 (pretreatment) and once weekly thereafter by BLI until animals became moribund. Bioluminescence in the untreated and imatinib groups continued to increase over time. In contrast, dasatinib-treated animals displayed a striking inhibition of tumor growth between pretreatment and end-of-drug treatment time points. Moreover, several animals showed regression of large primary tumors, as indicated by a decrease in bioluminescence signal (data not shown). Quantitative analysis of the mean photons emitted from the intracranial K562-pLUC\#2 tumors over time is shown in Figure 1A. Tumor bioluminescence increased significantly in the untreated control group. Imatinib treatment failed to inhibit intracranial K562-pLUC\#2 tumor growth, with results comparable with untreated controls, whereas dasatinib treatment resulted in complete regression of CNS tumors followed by tumor stasis while animals were on therapy. Antitumor activity, as determined by BLI, also correlated with increased survival. In contrast to imatinib, dasatinib had a significant effect on survival $(P<.05$ vs untreated control; Table 1$)$.

Experiment 3. To further delineate the effects of dasatinib, K562-pLUC\#2 cells were implanted and allowed to grow to well-established intracranial lesions. Dasatinib was then initiated at escalated doses of 25 or $50 \mathrm{mg} / \mathrm{kg}$ once daily for 30 days. Both dosages of dasatinib were associated with a significant increase in survival $(P<.001$ vs untreated control; Table 1$)$. Furthermore, BLI indicated that intracranial tumors regressed to near nondetectable levels and remained so as long as the animals were on treatment (Figures 1A,B and 2). Once drug administration stopped, all animals redeveloped intracranial tumors and eventually died.

To rationalize the robust antitumor activity of dasatinib in these intracranial CML models, we assessed the plasma and brain concentrations of dasatinib. Dasatinib brain concentrations were, on average, 12- to 31-fold lower than in plasma (ie, brain/plasma ratio or brain penetrance of $3.2 \%-8.6 \%$ ). But significantly, dasatinib pharmacokinetic analysis demonstrated that it readily achieved brain concentrations required to achieve $50 \%$ inhibition of cellular proliferation of K562 cells in vitro $(0.5 \mathrm{nM})$. For example, a daily dose of dasatinib $50 \mathrm{mg} / \mathrm{kg}$ was associated with brain concentrations of 10 to $100 \mathrm{nM}$ for nearly 24 hours (Figure 1C). This level of CNS penetration, based on this calculation, was sufficient to produce the antitumor activity observed in this study.

\section{Clinical effects of dasatinib in CNS manifestations of $\mathrm{Ph}^{+}$leukemia}

To investigate the potential clinical effects of dasatinib on CNS leukemia, we collected data from 14 patients with imatinibresistant or -intolerant $\mathrm{BC}-\mathrm{CML}$ or $\mathrm{Ph}^{+} \mathrm{ALL}$ and CNS involvement. Ten patients were enrolled in dasatinib clinical studies, whereas 4 patients received dasatinib for compassionate use. Of 


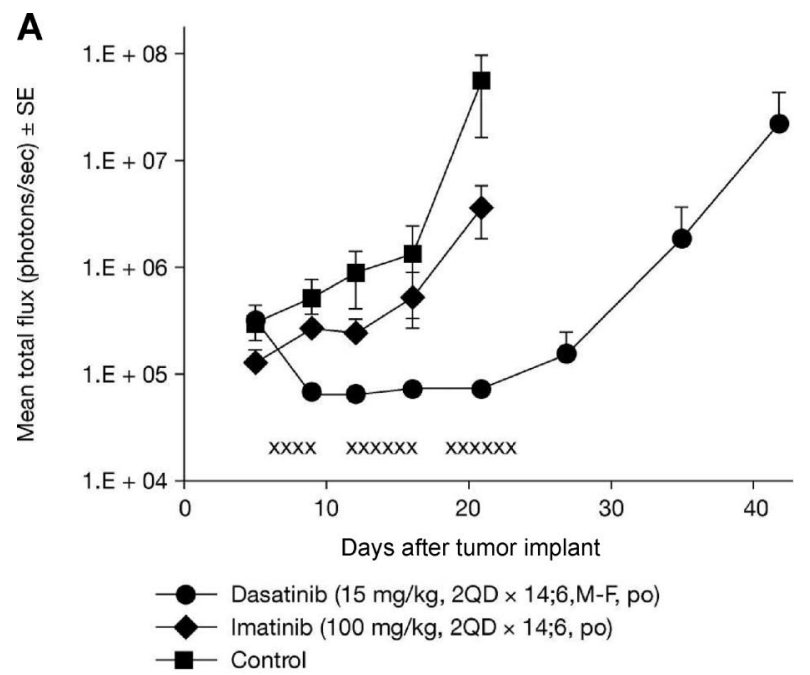

B
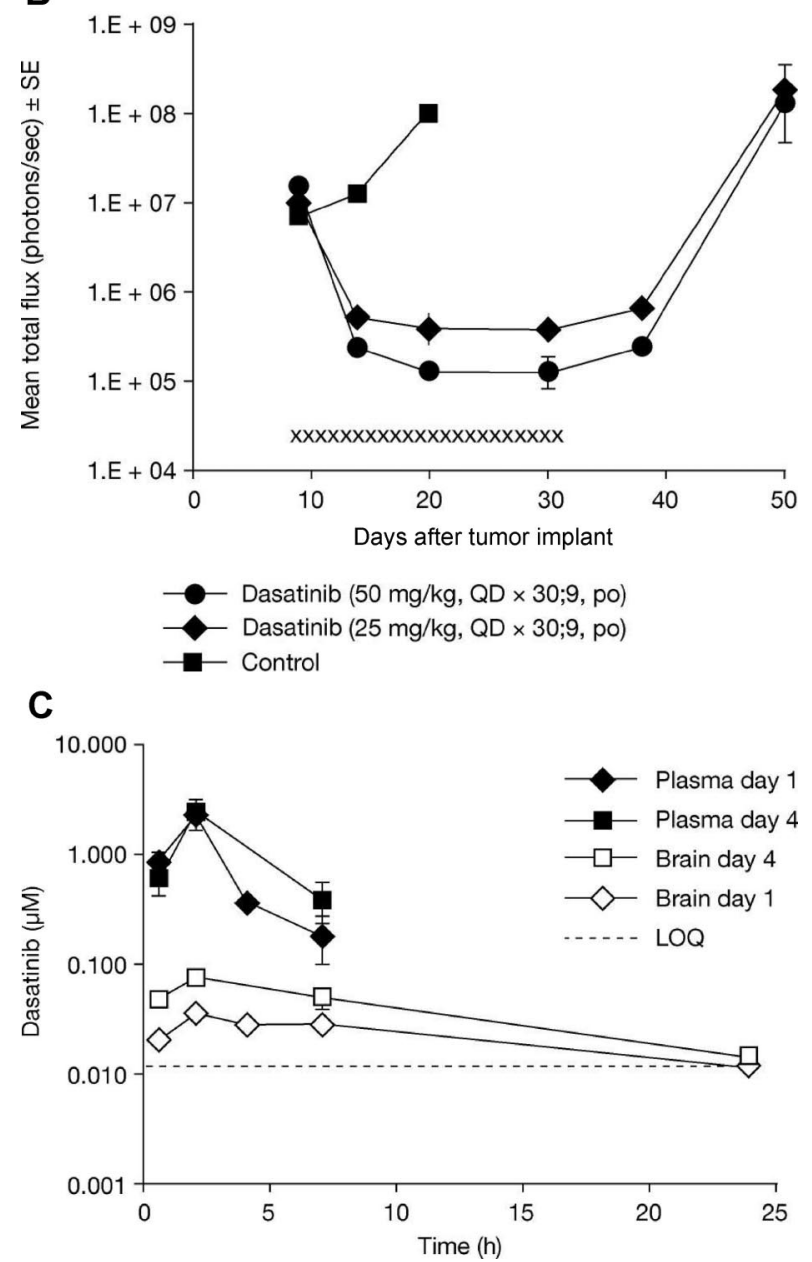

Figure 1. Comparative in vivo antitumor activity of dasatinib and imatinib in the intracranial K562-pLUC\#2 CML model and plasma/brain pharmacokinetic profile of dasatinib. (A) Tumor growth inhibition by dasatinib or imatinib in the K562-pLUC\#2 intracranial model as determined by BLI. Treatment was initiated on day 6 after tumor cell implantation. $\mathrm{x}$ indicates days of dasatinib administration. Mean photon emission plus or minus SE for each treatment set is shown ( $n=8-9$ mice per set). (B) Regression of large, well-established intracranial K562-pLUC\#2 tumors by dasatinib treatment as determined by BLI (study 3). Treatment was initiated on day 9 after tumor cell implantation. $\mathrm{x}$ indicates days of drug treatment. Mean photon emission plus or minus SE for each treatment set is shown ( $\mathrm{n}=8$ mice per set). (C) Plasma and brain pharmacokinetic profile for dasatinib. Each data point on the graph represents the average of 3 mice. Mice were killed at the indicated time, and plasma was prepared from whole blood obtained by cardiac puncture; brain biopsies were obtained from the same animals matching the plasma samples. these, 11 were treated with dasatinib as a first-line therapy for CNS leukemia, whereas 3 patients experienced a CNS relapse while on dasatinib therapy administered for other clinical reasons. Patient characteristics are described in Table 2, and treatment regimens are described in Table 3. Five patients received concomitant intrathecal therapy to treat CNS disease. The clinical course of an index patient with massive CNS involvement and dasatinib monotherapy is shown in Figure 3.

All of the 11 clinically evaluable patients responded to dasatinib (Table 3). Complete responses, defined as either disappearance of leukemic blasts from CSF or radiologic findings in magnetic resonance imaging, were observed in 7 patients. Four of these were achieved with dasatinib monotherapy. Of the 3 patients achieving a partial response, one had a very good partial response and 2 patients had long-lasting, clinically meaningful responses to dasatinib. One patient (patient 6) was only evaluated clinically for CNS disease and was considered to be stable at 22 months.

CNS responses to dasatinib were generally durable and were maintained for at least 6 months in 5 patients and for at least 12 months in 2 patients. Responses were maintained for at least 3 months in 9 evaluable patients.

To assess mechanisms of dasatinib resistance for CNS leukemia, we collected data from 3 additional patients who experienced a CNS relapse while on dasatinib. $B C R-A B L$ kinase domain sequencing from leukemic blast cells in CSF showed point mutations (T315I and V299L) in 2 patients conferring absolute resistance to dasatinib (no sequencing data available from one patient). Thus, CSF relapse was most likely attributed to selection of a dasatinib-resistant clone, not because of poor penetration of dasatinib into the CSF.

\section{Dasatinib concentrations in CSF}

CSF pharmacokinetic samples were obtained from a different group of patients: 15 adult subjects with CML or $\mathrm{Ph}^{+} \mathrm{ALL}$ and 7 pediatric subjects with $\mathrm{Ph}^{+}$ALL participating in dasatinib trials. The samples were collected approximately 3 hours after an oral dasatinib dose. Of these, detectable levels of dasatinib were found in only 2 of the adult patients ( 3.9 and $20.1 \mathrm{nM})$ and 4 of the pediatric patients $(1.4,1.9,2.3$, and $2.7 \mathrm{nM})$. For 3 of the 6 samples with detectable drug, CSF:plasma ratios could be determined $(0.05$, 0.08 , and 0.28). Levels of 2 metabolites were below the limit of quantification in all samples.

\section{Discussion}

We have shown here that dasatinib has antitumor effects in a mouse model of intracranial CML and that these benefits can be transferred to the clinic with dasatinib inducing substantial responses in patients with $\mathrm{CNS} \mathrm{Ph}^{+}$leukemia. In the mouse model, wellestablished intracranial CML tumors regressed with dasatinib treatment, significantly increasing animal survival. Moreover, complete disease stabilization was achieved for as long as animals were on dasatinib treatment. Hence, prolonged and continuous administration of this agent may be necessary to effectively control intracranial CML disease. Imatinib failed to show any activity in these tumor models, correlating with several clinical and preclinical findings. ${ }_{1,5,6,8,23}$ In addition to $B C R-A B L$, other mechanisms may be responsible for extramedullary disease expansion, including activation of Src family kinases. ${ }^{24-26}$ As well as its potent inhibitory effects on $B C R-A B L$, dasatinib also inhibits Src family 
Figure 2. Dasatinib induced regression of wellestablished intracranial K562-pLUC\#2 tumors in vivo. On day 9 after tumor implantation, animals with similarly well-established intracranial tumors were randomized into treatment and control sets $(n=8$ mice per set) and baseline images were taken $(A, C, E)$. Mice were then treated orally with either 50 or $25 \mathrm{mg} / \mathrm{kg}$ of dasatinib daily for 30 consecutive days. On day 20 after tumor implantation, a second set of bioluminescence images were taken $(B, D, F)$. (A,B) Dasatinib treatment, $50 \mathrm{mg} / \mathrm{kg}$. (C,D) Dasatinib treatment, $25 \mathrm{mg} / \mathrm{kg}$. $(E, F)$ Untreated control. Images were set at the same pseudo-color scale to show relative bioluminescent changes over time and signal intensity differences between treatment groups. Dasatinib treatment resulted in complete regression of intracranial tumors to near nondetectable levels, whereas untreated control showed progressive tumor growth with 2 of 8 animals dead on day 20 after tumor implant.
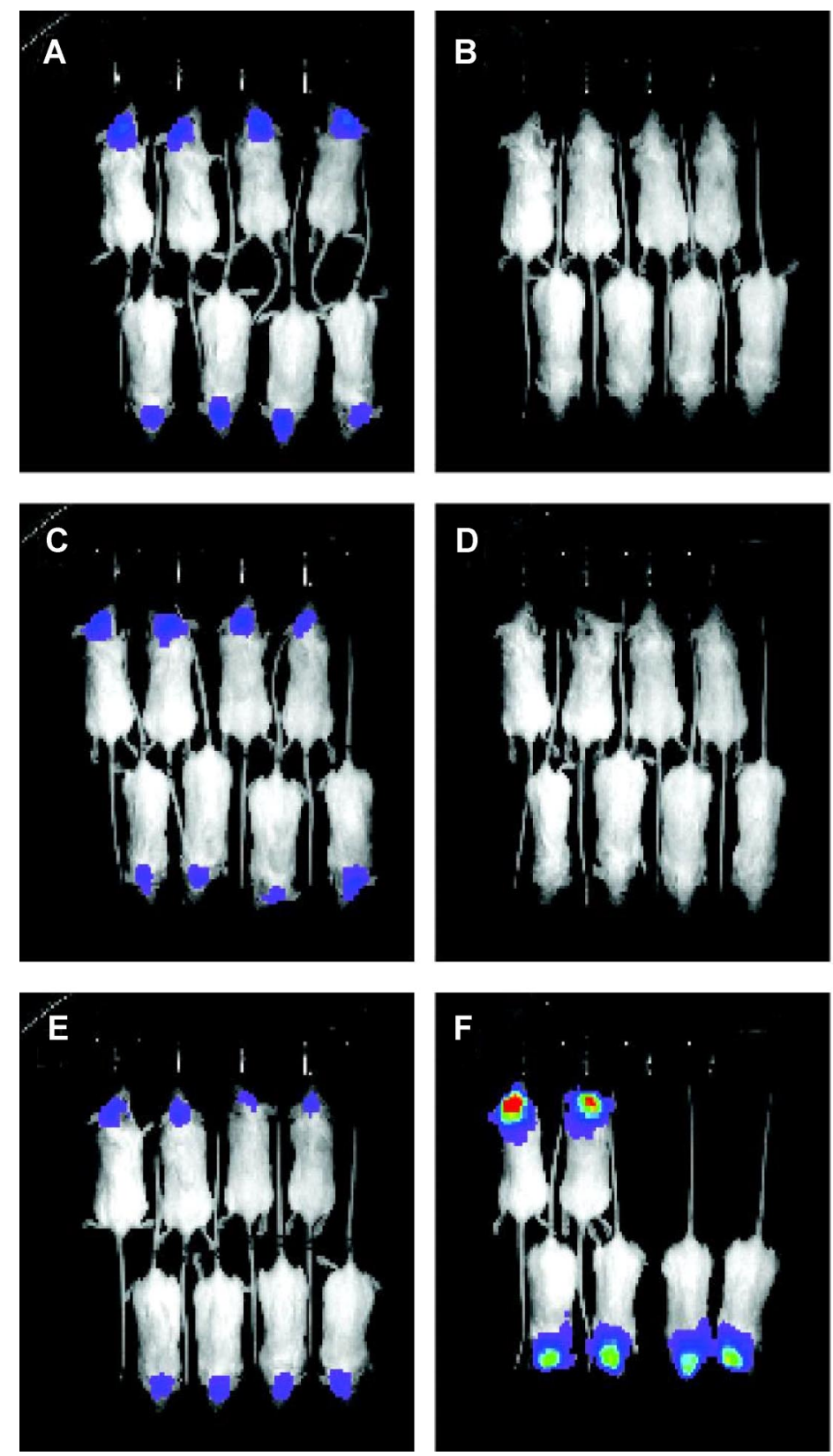

Photons/sec

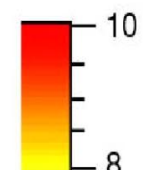

$\stackrel{x}{\vec{O}}$

Color bar

$\operatorname{Min}=1 . E+05$

$\operatorname{Max}=1 \cdot \mathrm{E}+07$ proteins, ${ }^{27}$ which may underlie the preclinical and clinical activity of dasatinib observed in our report.

During the absorptive phase (1-4 hours after dose), dasatinib concentrations in the brain were on average $5.5 \%$ of those found in the plasma (range, $3.2 \%-8.6 \%$ ) in mice. In the 3 patients for whom plasma and CSF concentrations were available, the brain penetrance was $5 \%, 8 \%$, and $28 \%$, respectively. This CNS penetration is considerably higher than that achieved by imatinib in several preclinical and clinical reports (brain penetrance ranging from $0.5 \%$ to $2 \%)^{1,2,5,6,8}$ It should be noted that the CNS penetrance of dasatinib is still low compared with other agents known to have good penetration of the blood-brain barrier, such as cytarabine. ${ }^{28}$

The extent to which increased blood-brain barrier penetrance by dasatinib versus imatinib is responsible for the apparent superior CNS antitumor effects of dasatinib is unclear. However, it is probable that other factors may also be important. For example, the much greater potency (325-fold) of dasatinib over imatinib ${ }^{15}$ may allow the achievement of a therapeutically effective concentration of dasatinib because it has been shown to be active at low or subnanomolar concentrations. ${ }^{15,16}$ This increased potency, along with the fact that the CSF is a low-protein environment where dasatinib is likely to exist as a free drug, suggests that even if dasatinib levels in the CNS are relatively low, the dasatinib concentrations achieved are sufficient for antitumor activity. The concentrations of dasatinib found in the CSF of patients (range, 1.4-20.1 nM) are consistent with the observed antitumor activity in the CNS. However, further studies are needed to determine the relationship of dasatinib CNS pharmacokinetics and clinical responses in more detail.

In these patients with $\mathrm{CNS} \mathrm{Ph}^{+}$leukemia, dasatinib was associated with substantial clinical responses. Our results confirm those of a single case report concerning the effects of dasatinib in $\mathrm{CNS} \mathrm{Ph}^{+}$leukemia. ${ }^{18}$ In the current study, clinically meaningful responses were achieved with dasatinib even as monotherapy and were maintained for more than or equal to 6 months in $45 \%$ of patients. Our data compare favorably with results from high-dose 
From www.bloodjournal.org at Erasmus MC Medical Library on May 20, 2009. For personal use only.

Table 2. Patient and disease characteristics

\begin{tabular}{|c|c|c|c|c|c|c|c|c|}
\hline $\begin{array}{l}\text { Patient } \\
\text { no. }\end{array}$ & Sex & Age, y & Disease & $\begin{array}{l}\text { Prior } \\
\text { SCT }\end{array}$ & CNS relapse site & $\begin{array}{c}\text { Isolated CNS relapse } \\
\text { (systemic disease status) }\end{array}$ & $\begin{array}{c}\text { Dasatinib as first-line } \\
\text { therapy for } \\
\text { CNS disease }\end{array}$ & $\begin{array}{c}\text { Enrolled into } \\
\text { dasatinib clinical } \\
\text { trials }\end{array}$ \\
\hline 1 & Male & 66 & $\mathrm{Ph}^{+} \mathrm{ALL}$ & No & CSF & No & Yes & Yes \\
\hline 2 & Male & 41 & Ly-BC-CML & Yes & CSF & Yes (stable MRD) & Yes & $\mathrm{No}^{*}$ \\
\hline 3 & Male & 7 & $\mathrm{Ph}^{+} \mathrm{ALL}$ & Yes & CSF & Yes (stable MRD) & Yes & Yes \\
\hline 4 & Male & 13 & $\mathrm{Ph}^{+} \mathrm{ALL}$ & Yes $(\times 2)$ & CSF & No & Yes & Yes \\
\hline 5 & Female & 59 & $\mathrm{BC}-\mathrm{CML}$ & Yes & Brain tumor (MRI) & Yes (CP) & Yes & $\mathrm{No}^{*}$ \\
\hline 6 & Male & 41 & My-BC-CML & No & Brain tumors (MRI) & No & Yes & Yes \\
\hline 7 & Female & 25 & $\mathrm{Ph}^{+} \mathrm{ALL}$ & No & CSF & Yes (stable MRD) & Yes & Yes \\
\hline 8 & Female & 35 & $\mathrm{Ph}+\mathrm{ALL}$ & Yes & CSF + brain tumors (MRI) & Yes (stable MRD) & Yes & Yes \\
\hline 9 & Male & 59 & My-BC-CML & Yes & CSF & Yes (stable MRD) & Yes & $\mathrm{No}^{*}$ \\
\hline 10 & Male & 12 & $\mathrm{Ph}^{+} \mathrm{ALL}$ & Yes & CSF & No & Yes & Yes \\
\hline 11 & Male & 10 & $\mathrm{Ph}^{+} \mathrm{ALL}$ & Yes $(\times 2)$ & CSF & No & Yes & Yes \\
\hline 12 & Male & 49 & $\mathrm{Ph}^{+} \mathrm{ALL}$ & Yes & CSF & Yes (stable MRD) & No & Yes \\
\hline 13 & Male & 79 & My-BC-CML & No & CSF & Yes (stable MRD) & No & Yes \\
\hline 14 & Male & 26 & Ly-BC-CML & No & CSF & Yes (CP) & No & $\mathrm{No}^{*}$ \\
\hline
\end{tabular}

SCT indicates stem cell transplantation; CNS, central nervous system; $\mathrm{Ph}^{+} \mathrm{ALL}$, Philadelphia chromosome-positive acute lymphoblastic leukemia; Ly-BC-CML, lymphoid blast crisis of CML; My-BC-CML, myeloid blast crisis of chronic myeloid leukemia; CSF, cerebrospinal fluid; MRI, magnetic resonance imaging; MRD, minimal residual disease; and $\mathrm{CP}$, chronic phase.

${ }^{\star}$ Enrolled in compassionate use programs.

cytarabine. ${ }^{29}$ Furthermore, the toxicity profile of oral dasatinib is superior to intensive intravenous chemotherapy.

In bone marrow $\mathrm{Ph}^{+}$leukemia, the most common mechanisms for dasatinib resistance are acquired $B C R-A B L$ point domain mutations in the gatekeeper position of the kinase (V299L, T315I, F317L). Of the 3 patients who experienced a CNS relapse on dasatinib, $B C R-A B L$ kinase domain mutation data from leukemic blasts in CSF was obtained for 2 patients. Both these patients showed selection of a dasatinibresistant leukemic clone in the CSF (V299L, T315I), which had caused disease relapse. This suggests a strong selection pressure exerted by dasatinib and thus access of the drug into the CSF.
Although chronic phase CML is derived from a malignant stem cell, the leukemia-initiating mutations in $\mathrm{Ph}^{+} \mathrm{ALL}^{30}$ and $\mathrm{CML}$ in blastic phase ${ }^{31}$ arise from a more committed progenitor. During dasatinib therapy, CNS relapse and expansion of a mutated clone in the CSF, but not in bone marrow, suggest that the origin of CNS leukemia is from a leukemic stem cell homing to CNS at the time of diagnosis. Theoretically, the CNS-specific homing of this leukemic stem cell prevents it from expanding outside of CNS. Interestingly, the CNS clone may be in a dormant state for many years as the time from diagnosis to CNS relapse in some of our patients was up to 8 years. Recent data indicate that cell surface receptors on leukemic

Table 3. Dasatinib treatment and response in evaluable patients

\begin{tabular}{|c|c|c|c|c|c|c|c|}
\hline $\begin{array}{l}\text { Patient } \\
\text { no. }\end{array}$ & Dasatinib dose & $\begin{array}{l}\text { Dose } \\
\text { regimen }\end{array}$ & $\begin{array}{c}\text { Dasatinib } \\
\text { treatment } \\
\text { duration, mo }\end{array}$ & $\begin{array}{l}\text { Concurrent } \\
\text { intrathecal } \\
\text { therapy }\end{array}$ & $\begin{array}{l}\text { Best } \\
\text { response }\end{array}$ & $\begin{array}{c}\text { Response } \\
\text { duration, mo }\end{array}$ & Therapy details \\
\hline 1 & $\begin{array}{l}140 \mathrm{mg} / \text { day, reduced due to } \\
\text { toxicity to } 50 \mathrm{mg} \times 5 \\
\text { days/week at } 8 \text { months }\end{array}$ & BID & 13 & No & CR & 11 & DXM at the beginning \\
\hline 2 & 140 mg/day & BID & $5+$ & Yes & CR & $5+$ & $\begin{array}{l}\text { IT therapy prior to start of dasatinib with } \\
\text { poor results, received three IT } \\
\text { therapies* after dasatinib start }\end{array}$ \\
\hline 3 & $\begin{array}{l}60 \mathrm{mg} / \mathrm{m}^{2} \text { ( } 25 \text { days) then } \\
80 \mathrm{mg} / \mathrm{m}^{2} \text { per day }\end{array}$ & QD & $10+$ & No & $\mathrm{CR}$ & 6 & $\begin{array}{l}\text { IT therapy initiated at } 6 \text { months for } \\
\text { relapse }\end{array}$ \\
\hline 4 & $\begin{array}{l}60 \mathrm{mg} / \mathrm{m}^{2} \text { (29 days) then } \\
80 \mathrm{mg} / \mathrm{m}^{2} \text { per day }\end{array}$ & QD & 4 & No & $\mathrm{CR}$ & 3 & $\begin{array}{l}\text { IT therapy initiated at } 3 \text { months due to } \\
\text { systemic relapse }\end{array}$ \\
\hline 5 & 140 mg/day & BID & $3+$ & No & Radiologic CR & $3+$ & None \\
\hline 6 & 140 mg/day & QD & $22+$ & No & Clinical CR & $22+$ & DXM at the beginning \\
\hline 7 & $\begin{array}{l}140 \mathrm{mg} / \text { day then } 200 \mathrm{mg} / \text { day at } \\
4 \text { months }\end{array}$ & BID & 21 & No & PR & 6 & $\begin{array}{l}\text { IT therapy and later cranial radiation } \\
\text { when treatment was intensified after } \\
6 \text { months due to relapse }\end{array}$ \\
\hline 8 & $\begin{array}{l}140 \mathrm{mg} / \text { day for } 3 \text { months, } \\
\text { varying dosing with long } \\
\text { interruptions, currently } 50 \mathrm{mg} \\
\text { every other day }\end{array}$ & QD & $26+$ & Yes & PR & $26+$ & DXM, IT therapy* $† \ddagger$ \\
\hline 9 & $140 \mathrm{mg} /$ day & BID & $3+$ & Yes & VGPR & $3+$ & IT therapy $†$ \\
\hline 10 & $60 \mathrm{mg} / \mathrm{m}^{2}$ per day & QD & 1.8 & Yes & CR & 1.5 & Systemic steroids, IT therapy†‡ \\
\hline 11 & $\begin{array}{l}80 \text { (39 days), } 120 \text { (20 days) } \\
\text { then } 160 \mathrm{mg} / \mathrm{m}^{2} \text { per day }\end{array}$ & BID & 2 & Yes & CR & 2 & IT therapy* \\
\hline
\end{tabular}

BID indicates twice daily; QD, once daily; CR, complete response; PR, partial response; VGPR, very good partial response; DXM, dexamethasone; and IT therapy, intrathecal therapy.

${ }^{\star}$ Cytarabine.

†Methotrexate.

†Prednisolone. 
Figure 3. Clinical efficacy of dasatinib monotherapy in the treatment of CNS leukemia in an index patient with relapsed $\mathrm{Ph}^{+}$ALL. CSF blast count was performed from May-Grünvald-Giemsa-stained cytospin slides by counting 100 to 300 cells. Microscopy slides were viewed with Zeiss Axioskop 40 light microscope (Carl Zeiss Microimaging, Jena, Germany) using Plan-Apochromat $63 \times / 1.4$ oil and Plan-Neofluor $100 \times / 1.3$ oil objective lenses. Images were acquired using Axiocam HRC digital camera (Carl Zeiss Microimaging) and were processed with Axiovision AC 4.2 software (Carl Zeiss Microimaging).

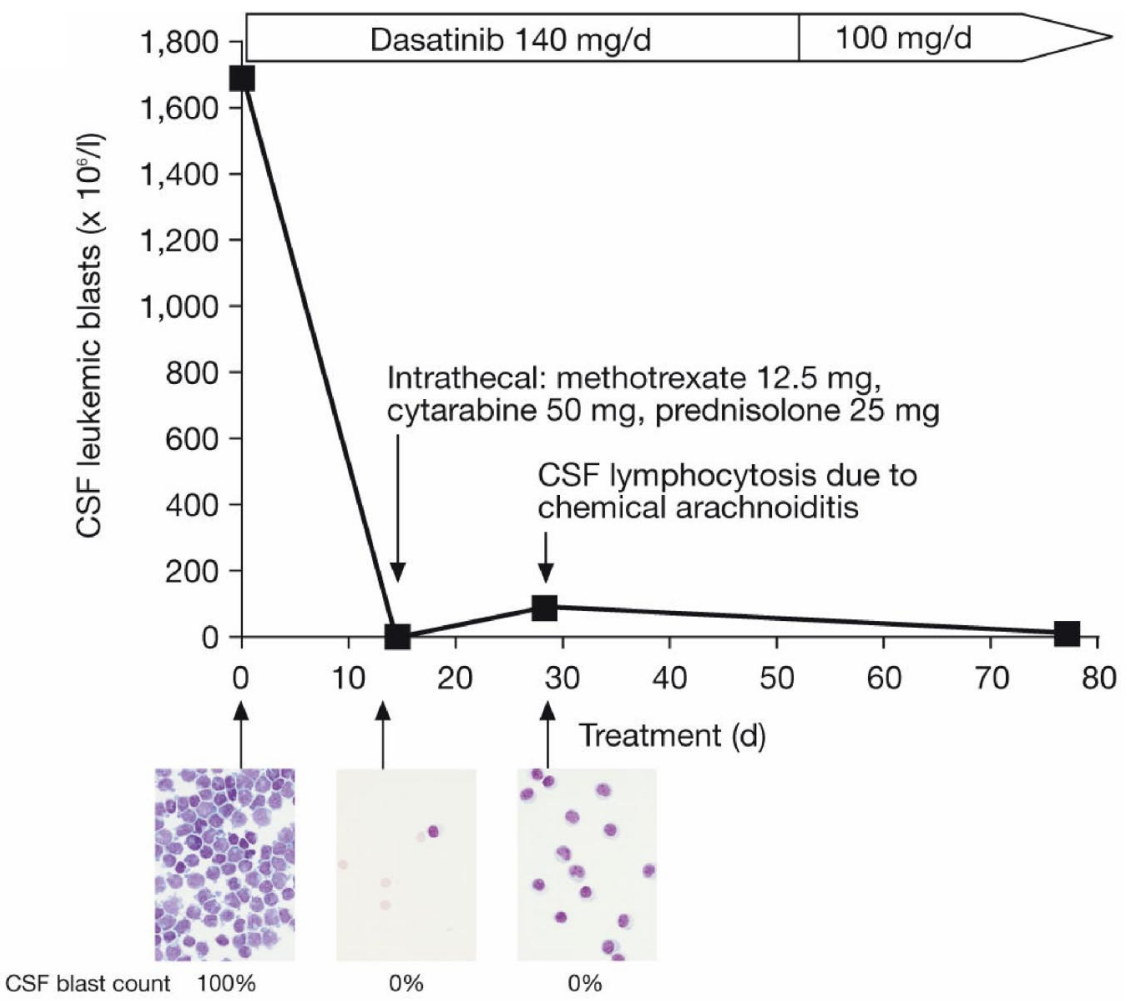

blasts, such as $\mathrm{CXCR} 4{ }^{32}$ or $\mathrm{CD} 44,{ }^{33}$ are key determinants in dissemination of leukemia to extramedullary sites. The exact molecular mechanisms are still to be defined but probably involve organ-specific "zip codes" on endothelial cells. ${ }^{34}$

In conclusion, dasatinib has promising therapeutic potential in managing intracranial leukemic disease, and substantial clinical activity in patients who experience CNS relapse while on imatinib therapy. Dasatinib may prove to be a valuable alternative to imatinib and an addendum to conventional cytotoxic therapy for $\mathrm{Ph}^{+} \mathrm{CNS}$ leukemia. Prospective, controlled studies are warranted to more fully determine treatment responses and characterize drug tolerability and safety in this patient population.

\section{Acknowledgments}

The authors thank Drs Robert Kramer, William Rose, and Roberto Weinmann for their critical comments on the preclinical work; Dr Rolf Ryseck for supplying the luciferase expression plasmid; Bengt Simonsson, Jens Stromsheim, and Lasse Ågren for help in the clinical case collection; Eeva Mäkinen, Minna Pajuportti, Christine Flefleh, Kelly McGlinchey, Krista Menard, and Amy Wiebesiek for excellent technical assistance; and Andrew Richardson (funded by Bristol-Myers Squibb) for editorial assistance.
This work was supported by the Finnish special governmental subsidy for health sciences, research and training, by the Finnish Cancer Societies (Helsinki, Finland), and the Finnish Association of Hematology (Hämeenlinna, Finland). Funding for preclinical studies and clinical trials was provided by Bristol-Myers Squibb (New York, NY).

\section{Authorship}

Contribution: K.P. initiated the clinical case collection studies; R.S., R.W., R.L., and F.Y.F.L. designed and performed preclinical studies; K.P., P.K., J.R., S.M., M.A., B.B., L.E., H.H.-H., M.H., H. Klamova, H. Knutsen, S.P., E.R., F.G., F.B.-B., H.D., E.E., R.F.D., R.P., and C.M.Z. performed clinical research; T.L. performed $B C R-A B L$ mutation analysis from CSF and blood samples; K.P. and F.Y.F.L. wrote the paper with contributions from the other authors; all authors approved the final version of the manuscript.

Conflict-of-interest disclosure: R.W. and R.L. were and R.S. and F.Y.F.L. are employed by Bristol-Myers Squibb, whose product was studied in the present work. The remaining authors declare no competing financial interests.

Correspondence: Kimmo Porkka, Hematology Research Unit, Biomedicum Helsinki, PO Box 700, FIN-00029 HUCH, Helsinki, Finland; e-mail: kimmo.porkka@ helsinki.fi.

\section{References}

1. Pfeifer $\mathrm{H}$, Wassmann B, Hofmann WK, et al. Risk and prognosis of central nervous system leukemia in patients with Philadelphia chromosomepositive acute leukemias treated with imatinib mesylate. Clin Cancer Res. 2003;9:4674-4681.

2. Leis JF, Stepan DE, Curtin PT, et al. Central nervous system failure in patients with chronic myelogenous leukemia lymphoid blast crisis and Philadelphia chromosome positive acute lympho- blastic leukemia treated with imatinib (STI-571). Leuk Lymphoma. 2004;45:695-698.

3. Matsuda M, Morita Y, Shimada T, et al. Extramedullary blast crisis derived from 2 different clones in the central nervous system and neck during complete cytogenetic remission of chronic myelogenous leukemia treated with imatinib mesylate. Int J Hematol. 2005;81:307-309.

4. Bujassoum S, Rifkind J, Lipton JH. Isolated cen- tral nervous system relapse in lymphoid blast crisis chronic myeloid leukemia and acute lymphoblastic leukemia in patients on imatinib therapy. Leuk Lymphoma. 2004;45:401-403.

5. Takayama N, Sato N, O'Brien SG, Ikeda Y, Okamoto S. Imatinib mesylate has limited activity against the central nervous system involvement of Philadelphia chromosome-positive acute lymphoblastic leukaemia due to poor penetration into 
From www.bloodjournal.org at Erasmus MC Medical Library on May 20, 2009. For personal use only.

cerebrospinal fluid. Br J Haematol. 2002;119:106108.

6. Dai $H$, Marbach $P$, Lemaire $M$, Hayes $M$, Elmquist WF. Distribution of STI-571 to the brain is limited by P-glycoprotein-mediated efflux. J Pharmacol Exp Ther. 2003;304:1085-1092.

7. Senior K. Gleevec does not cross blood-brain barrier. Lancet Oncol. 2003;4:198.

8. Bornhauser M, Jenke A, Freiberg-Richter J, et al. CNS blast crisis of chronic myelogenous leukemia in a patient with a major cytogenetic response in bone marrow associated with low levels of imatinib mesylate and its $\mathrm{N}$-desmethylated metabolite in cerebral spinal fluid. Ann Hematol. 2004;83:401-402.

9. Cortes J. Central nervous system involvement in adult acute lymphocytic leukemia. Hematol Oncol Clin North Am. 2001;15:145-162.

10. Hochhaus A, Kantarjian HM, Baccarani M, et al. Dasatinib induces notable hematologic and cyto genetic responses in chronic-phase chronic myeloid leukemia after failure of imatinib therapy. Blood. 2007;109:2303-2309.

11. Cortes J, Rousselot P, Kim DW, et al. Dasatinib induces complete hematologic and cytogenetic responses in patients with imatinib-resistant or -intolerant chronic myeloid leukemia in blast crisis. Blood. 2007;109:3207-3213.

12. Guilhot F, Apperley J, Kim DW, et al. Dasatinib induces significant hematologic and cytogenetic responses in patients with imatinib-resistant or -intolerant chronic myeloid leukemia in accelerated phase. Blood. 2007; 109:4143-4150.

13. Kantarjian $\mathrm{H}$, Pasquini $\mathrm{R}$, Hamerschlak N, et al. Dasatinib or high-dose imatinib for chronic-phase chronic myeloid leukemia after failure of first-line imatinib: a randomized phase 2 trial. Blood. 2007; 109:5143-5150.

14. Ottmann O, Dombret H, Martinelli G, et al. Dasatinib induces rapid hematologic and cytogenetic responses in adult patients with Philadelphia chromosome positive acute lymphoblastic leukemia with resistance or intolerance to imatinib: interim results of a phase 2 study. Blood. 2007;110: 2309-2315.

15. O'Hare T, Walters DK, Stoffregen EP, et al. In vitro activity of Bcr-Abl inhibitors AMN107 and
BMS-354825 against clinically relevant imatinibresistant Abl kinase domain mutants. Cancer Res. 2005;65:4500-4505.

16. Shah NP, Tran C, Lee FY, Chen P, Norris D, Sawyers CL. Overriding imatinib resistance with a novel ABL kinase inhibitor. Science. 2004;305: 399-401.

17. Lee FY, Wen ML, Bhide R, et al. Dasatinib (BMS354825) overcomes multiple mechanisms of imatinib resistance in chronic myeloid leukemia (CML). Blood. 2005;106: Abstract 1994.

18. Abdelhalim A, Barcos M, Block AW, et al. Remission of Philadelphia chromosome-positive central nervous system leukemia after dasatinib therapy. Leuk Lymphoma. 2007;48:1053-1056.

19. Odom LF, Wilson H, Cullen J, Bank J, Blake M, Jamieson B. Significance of blasts in low-cellcount cerebrospinal fluid specimens from children with acute lymphoblastic leukemia. Cancer. 1990; 66:1748-1754.

20. Gabert J, Beillard E, van der Velden VH, et al. Standardization and quality control studies of "real-time" quantitative reverse transcriptase polymerase chain reaction of fusion gene transcripts for residual disease detection in leukemia: a Europe Against Cancer program. Leukemia. 2003:17:2318-2357.

21. Branford S, Rudzki Z, Walsh S, et al. High frequency of point mutations clustered within the adenosine triphosphate-binding region of $\mathrm{BCR} /$ $A B L$ in patients with chronic myeloid leukemia or Ph-positive acute lymphoblastic leukemia who develop imatinib (STI571) resistance. Blood. 2002;99:3472-3475.

22. Soverini S, Martinelli G, Amabile M, et al. Denaturing-HPLC-based assay for detection of ABL mutations in chronic myeloid leukemia patients resistant to Imatinib. Clin Chem. 2004:50: 1205-1213.

23. Wolff NC, Richardson JA, Egorin M, llaria RL Jr. The CNS is a sanctuary for leukemic cells in mice receiving imatinib mesylate for $\mathrm{Bcr} / \mathrm{Abl}$-induced leukemia. Blood. 2003;101:5010-5013.

24. Donato NJ, Wu JY, Stapley J, et al. BCR-ABL independence and LYN kinase overexpression in chronic myelogenous leukemia cells selected for resistance to STI571. Blood. 2003;101:690-698.

25. Dai Y, Rahmani M, Corey SJ, Dent P, Grant S. A
Bcr/Abl-independent, Lyn-dependent form of imatinib mesylate (STI-571) resistance is associated with altered expression of Bcl-2. J Biol Chem. 2004;279:34227-34239.

26. Ptasznik A, Nakata Y, Kalota A, Emerson SG, Gewirtz AM. Short interfering RNA (siRNA) targeting the Lyn kinase induces apoptosis in primary, and drug-resistant, BCR-ABL1(+) leukemia cells. Nat Med. 2004;10:1187-1189.

27. Lombardo LJ, Lee FY, Chen P, et al. Discovery of $\mathrm{N}$-(2-chloro-6-methyl-phenyl)-2-(6-(4-(2-hydroxyethyl)-piperazin-1-yl)-2-methylpyrimidin-4ylamino)thiazole-5-carboxamide (BMS-354825), a dual Src/Abl kinase inhibitor with potent antitumor activity in preclinical assays. J Med Chem. 2004;47:6658-6661

28. Slevin ML, Piall EM, Aherne GW, Johnston A, Lister TA. The pharmacokinetics of cytosine arabinoside in the plasma and cerebrospinal fluid during conventional and high-dose therapy. Med Pediatr Oncol. 1982;10(suppl 1):157-168.

29. Morra E, Lazzarino M, Brusamolino E, et al. The role of systemic high-dose cytarabine in the treatment of central nervous system leukemia: clinical results in 46 patients. Cancer. 1993;72:439-445

30. Castor A, Nilsson L, Astrand-Grundstrom I, et al Distinct patterns of hematopoietic stem cell involvement in acute lymphoblastic leukemia. Nat Med. 2005;11:630-637.

31. Jamieson $\mathrm{CH}$, Ailles LE, Dylla SJ, et al. Granulocyte-macrophage progenitors as candidate leukemic stem cells in blast-crisis CML. N Engl J Med. 2004;351:657-667.

32. Burger JA, Burkle A. The CXCR4 chemokine receptor in acute and chronic leukaemia: a marrow homing receptor and potential therapeutic target. Br J Haematol. 2007;137:288-296.

33. Krause DS, Lazarides $\mathrm{K}$, von Andrian UH, Van Etten RA. Requirement for CD44 in homing and engraftment of BCR-ABL-expressing leukemic stem cells. Nat Med. 2006;12:1175-1180.

34. Porkka K, Laakkonen P, Hoffman JA, Bernasconi $M$, Ruoslahti E. A fragment of the HMGN2 protein homes to the nuclei of tumor cells and tumor endothelial cells in vivo. Proc Natl Acad Sci U S A. 2002;99:7444-7449. 